\title{
Pancreatic stenting to prevent post-ERCP pancreatitis: a randomized multicenter trial
}

\section{(ㄷ)(1) $\odot$}

\section{Authors}

Veit Phillip ${ }^{1}$, Aldis Pukitis², Alexey Epstein ${ }^{3}$, Alexander Hapfelmeier ${ }^{4}$, David Haf ${ }^{1}$, Miriam Schwab ${ }^{1}$, Ihsan Ekin Demir ${ }^{5}$, Jonas Rosendahl ${ }^{6}$, Albrecht Hoffmeister ${ }^{6}$, Roland M. Schmid ${ }^{1}$, Andreas Weber ${ }^{1}$, Hana Algül ${ }^{1}$

Institutions

1 Klinik und Poliklinik für Innere Medizin II, Klinikum rechts der Isar, Technische Universität München, München, Germany

2 Pauls Stradins Clinical University Hospital, Center of Gastroenterology, Hepatology and Nutrition, Riga, Latvia

3 First Clinical Hospital named after E.E. Volosevich, Arkhangelsk, Russia

4 Institut für Medizinische Informatik, Statistik und Epidemiologie, Klinikum rechts der Isar, Technische Universität München, München, Germany

5 Klinik und Poliklinik für Chirurgie, Klinikum rechts der Isar, Technische Universität München, München, Germany

6 Klinik für Gastroenterologie und Rheumatologie, Department für Innere Medizin, Neurologie und Dermatologie, Universitätsklinikum Leipzig, Leipzig, Germany

submitted 4.9.2018

accepted after revision 29.1.2019

Bibliography

DOI https://doi.org/10.1055/a-0886-6384 |

Endoscopy International Open 2019; 07: E860-E868

(c) Georg Thieme Verlag KG Stuttgart · New York eISSN 2196-9736

Corresponding author

Prof. Dr. Hana Algül, MPH, Klinikum rechts der Isar, Technische Universität München, Klinik und Poliklinik für Innere Medizin II, Ismaningerstr. 22, 81675 München, Germany

Fax: $+49089-4140-4115$

hana.alguel@mri.tum.de

\section{ABSTRACT}

Background and study aims Acute pancreatitis (AP) is one of the most common gastrointestinal disorders leading to hospitalization and the most frequent complication of endoscopic retrograde cholangiopancreatography (ERCP). Besides pharmaco-prophylaxis, pancreatic stenting has been demonstrated to protect from post-ERCP pancreatitis (PEP). However, it remains unclear which patients benefit from pancreatic stenting. We therefore hypothesized that in an unselected population, inadvertent cannulation of the pancreatic duct during first-time ERCP increases risk of PEP and that this risk can be significantly reduced by pancreatic stenting.

Patients and methods This study was a multicenter, prospective, randomized controlled trial conducted at four European centers. A total of 167 patients undergoing firsttime ERCP were enrolled in this trial. In the case of inadvertent cannulation of the pancreatic duct, patients were randomly assigned to receive either a 5 French plastic pancreatic stent of various length or no routine prophylactic intervention for PEP.

Results A total of 167 patients were included in the final analysis. Prophylactic stent insertion significantly reduced the rate of PEP during first-time ERCP (odds ratio 0.43; $95 \%$ confidence interval $0.19-0.98 ; P=0.04)$. The number needed to treat to prevent one case of PEP by prophylactic stent insertion after inadvertent cannulation of the pancreatic duct was 8.1 for the intention-to-treat population. Conclusion In an unselected patient population, inadvertent cannulation of the pancreatic duct during first-time ERCP is associated with a high risk for PEP. This risk can be significantly reduced by prophylactic pancreatic stenting, which is a safe and feasible procedure.

\section{Introduction}

Acute pancreatitis (AP) is one of the most common gastrointestinal disorders leading to hospitalization in the United States [1]. In about $80 \%$ of all cases, AP has an alcoholic or biliary etiol- ogy [2]. In patients requiring endoscopic retrograde cholangiopancreatography (ERCP), post-ERCP pancreatitis (PEP) is the most common adverse event that, at the very minimum, prolongs the duration of the hospital stay and, in rare cases, causes 
serious morbidity and death [3]. The rate of PEP is about $3.5 \%$ of all ERCP procedures, however, the rate can increase to more than $25 \%$ in high-risk patients [3,4]. Although diagnostic ERCP has been largely replaced by other procedures such as magnetic resonance cholangiopancreatography (MRCP) and endoscopic ultrasound (EUS), it is still an indispensable therapeutic tool for patients with many common diseases such as gallstone-induced obstructive cholangitis. As there is no causal and efficacious therapy for pancreatitis, prevention of PEP is a crucial goal of overall management of these patients.

Several studies have identified risk factors for PEP and some have further aimed to reduce the rate of PEP utilizing various approaches [5]. The most promising prophylactic interventions include anti-inflammatory drugs and pancreatic duct stents. Although several studies have shown that non-steroidal anti-inflammatory drugs (NSAID) reduce risk of PEP and routine rectal administration of $100 \mathrm{mg}$ of diclofenac or indomethacin is recommended by the European Society of Gastrointestinal Endoscopy (ESGE) for prophylaxis of PEP, these data have been challenged by other randomized trials [5,6]. Of note, all but one study supporting the recommendation of prophylactic NSAID administration included only high-risk patients, and only the study by Luo et al. probably represented the cross-section of ERCPs typically performed in the United States [7, 8]. In addition to use of prophylactic drugs, ESGE guidelines recommend strongly considering insertion of a prophylactic pancreatic stent in patients considered to be at high risk of PEP. Once again, studies that have led to this recommendation are heterogeneous and nearly all of them are limited to high-risk patients, while the type of risk factors for PEP differed between the studies $[9,10]$.

One of the described procedure-related risk factors is inadvertent cannulation of the pancreatic duct, which may lead to swelling with outflow obstruction and consecutive PEP [5,7]. In this situation, pancreatic duct stenting will guarantee unhindered drainage of pancreatic secretions and might reduce the rate of PEP.

Our aim was to investigate the effect of prophylactic pancreatic duct stenting in an unselected, cross-sectional patient population undergoing first-time ERCP with inadvertent cannulation of the pancreatic duct. Therefore, we performed a multicenter, prospective, randomized trial entitled Post-ERCP pancreatitis Prevention by Stent Insertion (PEPSI).

\section{Patients and methods}

\section{Study design}

This study was a multicenter, prospective, randomized controlled trial conducted between July 2010 and April 2016at four European tertiary referral centers within the Pancreas 2000 collaborative program [11]. The study was conducted in accordance with the Declaration of Helsinki and was approved by the local ethics committees (project number 2625/09). The study was also registered at Clinicaltrials.gov (ClinicalTrials.gov Identifier: NCT01673763)

\section{Patients}

All patients undergoing first-time ERCP during the study period at one of the four participating university-affiliated centers were eligible for study inclusion. Patients were not enrolled in the study if they had AP at the time of ERCP or there was an intention to cannulate the pancreatic duct. Further, patients younger than 18 years or those that were pregnant were not enrolled into the study.

\section{Intervention}

Eligible patients who provided written informed consent and in whom the pancreatic duct was inadvertently cannulated underwent randomization. Randomization was provided centrally by the Institute of Medical Informatics, Statistics and Epidemiology of the Technische Universität München. Randomization was stratified by center using varying block sizes and was sent out in sealed envelopes. All procedure-related interventions were dictated by the performing endoscopist. Guidewire-assisted standard technique for biliary cannulation was primarily applied. However, pancreatic guidewire-assisted biliary cannulation, needle-knife fistulotomy, and transpancreatic biliary sphincterotomy were also allowed according to the endoscopists' choice. In the case of inadvertent cannulation of the pancreatic duct, patients were randomly assigned to receive either a 5 French plastic stent (mandel+rupp medizintechnik gmbh, 40699 Erkrath, Germany) of various length determined by the local endoscopist or no prophylactic measure for PEP. Inadvertent cannulation was defined as any cannulation of the pancreatic duct by wire, papillotome or huibregtse. Levels of difficulty for cannulation of the papilla duodeni major were categorized as easy ( $1-5$ attempts), moderate ( $6-10$ attempts), or difficult (> 10 attempts) as determined by the endoscopist. The pancreatic stent was endoscopically removed 3 to 5 days after ERCP, if it had not spontaneously dislodged in the interim. Data were collected at the participating centers and were combined using an internet-based database.

\section{Sample size and power calculation}

Assuming a reduction in incidence from $20 \%$ to $5 \%$ [12, 13] for post-ERCP pancreatitis by stenting the pancreatic duct (referring to post-ERCP follow-up period of 5 days), the required sample size was 82 patients per group to establish this clinically relevant difference with $80 \%$ power by employing a Fisher's exact test at a two-sided level of significance of $5 \%$ (nQuery Advisor 7.0$)$.

\section{Study outcome}

The primary endpoint of the study was development of PEP, which was diagnosed according to the revised Atlanta Classification if two out of the following three criteria were present: new onset of pain in the upper abdomen, an elevation in serum activities of pancreatic enzymes of at least three times the upper limit of the reference interval on the day after ERCP, or imaging findings of AP. The assessor was blinded to the treatment. Secondary endpoint was the severity of PEP as defined by the revised Atlanta classification [14]. 


\section{Statistical analyses}

Statistical analyses were performed using IBM SPSS Statistics 24 (IBM Corp., Armonk, New York, United States).

For the primary endpoint, development of PEP was described by absolute and relative frequencies and was compared between treatment groups using a chi-square test on a confirmatory two-sided $5 \%$ significance level.

For secondary endpoints, mean \pm standard deviation and median (range; interquartile range [IQR]) were used as descriptive statistics of normally and non-normally distributed data, respectively. Hypothesis testing of group differences was performed by Mann-Whitney-U tests. Distribution of categorical data were presented by absolute and relative frequencies. Corresponding hypothesis testing was conducted by use of chisquare tests or a Fisher's exact test.

Multivariable analyses for adjusted effect estimation and investigation of interaction effects were performed by binary logistic regression. To ensure consistent effect estimation, these models were restricted to a 1:10 ratio of estimated model parameters to the size of the less frequent outcome class. Here, PEP occurred in 31 of 167 patients which enabled estimation of $31 / 10 \approx 3$ parameters [15]. The odds ratio served as corresponding effect measure. Hypothesis testing was performed on exploratory, two-sided $5 \%$ significance levels.

In two patients randomized for a prophylactic stent placement, insertion of the stent was not successful due to technical difficulties. In both patients, mild PEP was documented. Ac- cording to the guidelines of the International Council for Harmonisation of Technical Requirements for Pharmaceuticals for Human Use $(\mathrm{ICH})$, these patients were excluded from the analyses [16].

Confidence intervals for the number needed to treat (NNT) are based on Wilson score intervals without continuity correction [17].

All authors had access to the study data and reviewed and approved the final manuscript.

\section{Results}

\section{Patients}

In total 167 patients were included in the final analysis. Prophylactic pancreatic stenting was performed in 87 (52.1\%) patients in which the pancreatic duct was inadvertently cannulated, while in 80 (47.9\%) no stenting was undertaken. Baseline characteristics demonstrated no relevant differences between the two groups ( $\triangleright$ Table 1 ). A follow-up of all patients was completed.

\section{Study outcomes}

The primary outcome of PEP occurred in 31 of 167 patients (18.6\%). In 29 of 31 cases, (93.5\%) the classification was mild pancreatitis and in two cases (6.5\%), the classification was moderately severe pancreatitis. PEP was diagnosed in 11 of 87 patients $(12.6 \%)$ in the pancreatic stent group and in 20 of 80 patients $(25.0 \%)$ in the non-stent group, corresponding to a

- Table 1 Patient characteristics.

\begin{tabular}{|c|c|c|c|}
\hline & All patients & Stent group & No stent group \\
\hline Sex, female & $96 / 167(57 \%)$ & $55 / 87(63 \%)$ & $41 / 80(51 \%)$ \\
\hline Age, years & $60.7 \pm 14.5$ & $59.8 \pm 15.5$ & $61.6 \pm 13.2$ \\
\hline Body weight, $\mathrm{kg}$ & $\begin{array}{l}74.0 \\
(45-130 ; 66-83)\end{array}$ & $\begin{array}{l}75.0 \\
(45-109 ; 67-84)\end{array}$ & $\begin{array}{l}73.5 \\
(48-130 ; 65.8-81.3)\end{array}$ \\
\hline Body height, $\mathrm{cm}$ & $169.9 \pm 8.8$ & $169.9 \pm 7.9$ & $169.8 \pm 9.7$ \\
\hline $\mathrm{BMI}, \mathrm{kg} / \mathrm{m}^{2}$ & $\begin{array}{l}25.5 \\
(16.7-39.6 ; 23.5-28.6)\end{array}$ & $\begin{array}{l}25.6 \\
(16.7-39.6 ; 23.5-29)\end{array}$ & $\begin{array}{l}25.5 \\
(18.1-38.8 ; 23.4-27.5)\end{array}$ \\
\hline Bile duct diameter, $\mathrm{mm}$ & $\begin{array}{l}10 \\
(3-37 ; 8-14)\end{array}$ & $\begin{array}{l}10 \\
(4-37 ; 7-14)\end{array}$ & $\begin{array}{l}11 \\
(3-25 ; 8-15)\end{array}$ \\
\hline Serum bilirubin, mg/dL & $\begin{array}{l}3.3 \\
(0.2-36.1 ; 0.9-7.6)\end{array}$ & $\begin{array}{l}4.0 \\
(0.2-28.4 ; 0.9-9.3)\end{array}$ & $\begin{array}{l}2.7 \\
(0.2-36.1 ; 0.9-6.4)\end{array}$ \\
\hline \multicolumn{4}{|l|}{ Diagnosis } \\
\hline Bile duct stones & $69 / 167(41.3 \%)$ & $31 / 87(35.6 \%)$ & $38 / 80(47.5 \%)$ \\
\hline Jaundice of unknown origin & $10 / 167(6.0 \%)$ & $7 / 87(8.0 \%)$ & $3 / 80(3.8 \%)$ \\
\hline Benign bile duct stricture & $24 / 167(14.4 \%)$ & $13 / 87(14.9 \%)$ & $11 / 80(13.8 \%)$ \\
\hline Malignant bile duct stricture & $42 / 167(25.1 \%)$ & $24 / 87(27.6 \%)$ & $18 / 80(22.5 \%)$ \\
\hline Unknown bile duct stricture & $16 / 167(9.6 \%)$ & $9 / 87(10.3 \%)$ & $7 / 80(8.8 \%)$ \\
\hline
\end{tabular}




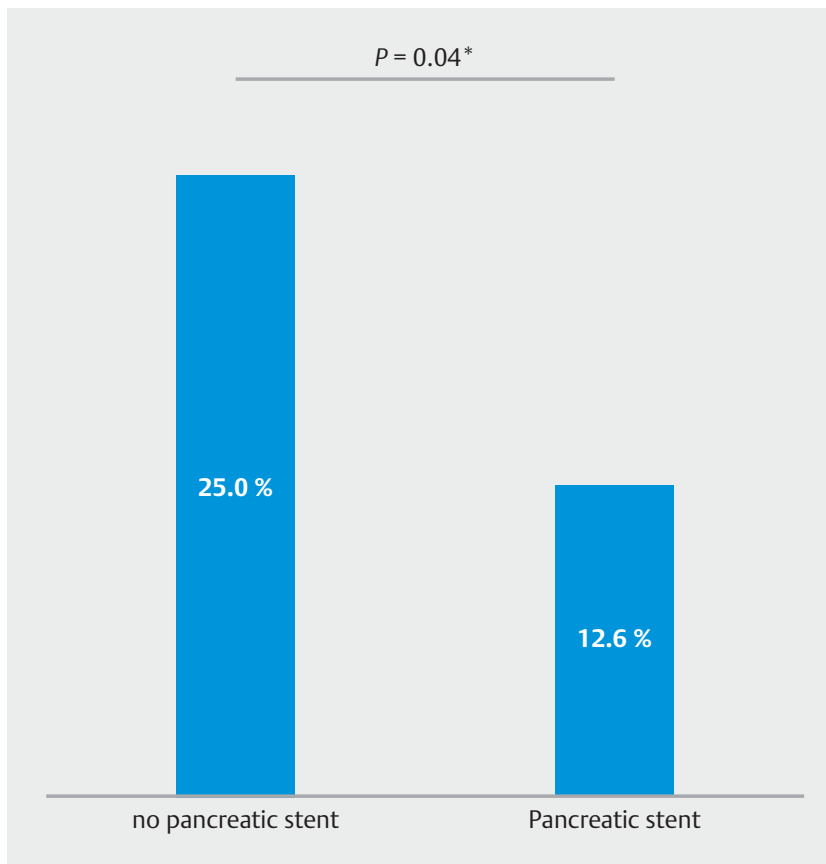

- Fig. 1 Bar graph showing the rate of post-ERCP pancreatitis (PEP) in patients without prophylactic measurements for PEP and $\mathrm{pa}$ tients receiving a prophylactic pancreatic stent (Odds ratio 0.434 $95 \%$ confidence interval $0.19-0.98 ; P=0.04)$.

statistically significant reduction of PEP by prophylactic stent insertion (odds ratio (OR) 0.43; $95 \%$ confidence interval (95\% Cl) $0.19-0.98 ; P=0.04$; Fig. 1 ).

The number needed to treat to prevent one case of PEP by prophylactic stent insertion after inadvertent cannulation of the pancreatic duct was 8.1 for the intention-to-treat (ITT) population. After exclusion of the two patients with unsuccessful stent placement, the number needed to treat was 7.0 for the full analysis set. There was no considerable influence of the length of the pancreatic stent on risk of developing PEP (OR 1.06; $95 \% \mathrm{Cl} 0.74-1.51) ; P=0.75)$.

\section{Patient and procedure-related risk factors for PEP}

Univariable analysis did not reveal risk factors related to patient characteristics or indications for ERCP ( $\triangleright$ Supplementary Table 1). A multivariable analysis, including the highly suspected risk factors "female sex" and "young age" (i.e., <40 years) as well as the risk-reducing pancreatic stent demonstrated a suggestive increased risk for patients younger than 40 years (OR 3.89; $95 \% \mathrm{Cl} 0.96$ - 15.73; $P=0.06$; Fig. 2a).

In addition, multivariable analysis including the earlier described patient-related risk factors non-dilated bile duct, nonelevated serum bilirubin, and the risk-reducing pancreatic stent itself revealed a non-dilated bile duct as a potential, independent risk factor for PEP (OR 2.63; $95 \% \mathrm{Cl} 1.00$ - 5.84; $P=0.06$;

\section{> Fig. 2b).}

For diagnosis-related risk factors, there was no crucial association in the univariable analysis. The same applied to the multivariable analysis that included the clinically suspected risk factors "bile duct stones" and "bile duct strictures" as well as the

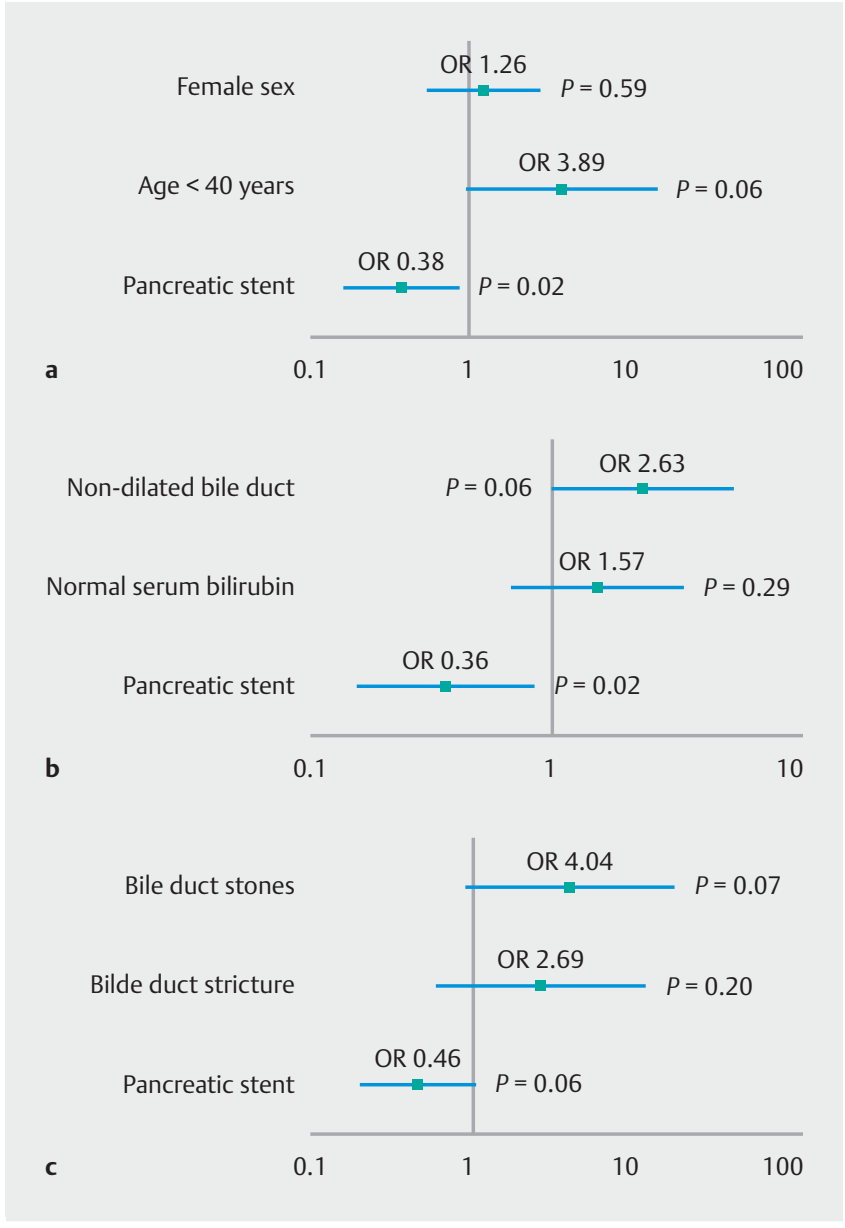

- Fig. 2 a Forest plot showing risk factors for post-ERCP pancreatitis. Black squares indicate the odds ratio (OR), lines represent the $95 \%$ confidence interval. b Forest plot showing risk factors for post-ERCP pancreatitis. Black squares indicate the odds ratio (OR), lines represent the $95 \%$ confidence interval. c Forest plot showing risk factors for post-ERCP pancreatitis. Black squares indicate odds ratio (OR), lines represent the $95 \%$ confidence interval.

risk-reducing pancreatic stent. Both risk factors did not show a statistically significant increase in risk of PEP (bile duct stones, OR 4.04; $95 \% \mathrm{Cl} 0.89-18.25 ; P=0.07$; bile duct strictures, $O R$ 2.69; $95 \% \mathrm{Cl} 0.59-12.21 ; P=0.20$; Fig. 2c).

However, several procedure-related risk factors were statistically significantly associated with PEP in univariable analysis ( Supplementary Table 1). In summary, there was only a negligible increased risk for PEP after cannulation of the bile duct by any wire (OR 1.16; $95 \% \mathrm{Cl} 0.39-3.68 ; P=0.80)$.

Regarding the pancreatic duct, cannulation using a Terumo wire (OR 5.36; $95 \% \mathrm{Cl} 1.94-14.77 ; P<0.01$ ) or a papillotome (OR 2.88; $95 \% \mathrm{Cl} 1.20-6.88 ; P=0.02$ ) significantly increased risk of developing PEP in the univariable analysis ( Supplementary Table 1 ).

Cannulation of the pancreatic duct using a Terumo wire was associated with difficult cannulation of the papilla (>10 attempts; OR 2.64; $95 \%$ Cl 1.34-5.21; P<0.01). Regarding PEP, cannulation of the pancreatic duct using a Terumo wire, but not the difficult cannulation of the papilla, was an independent 


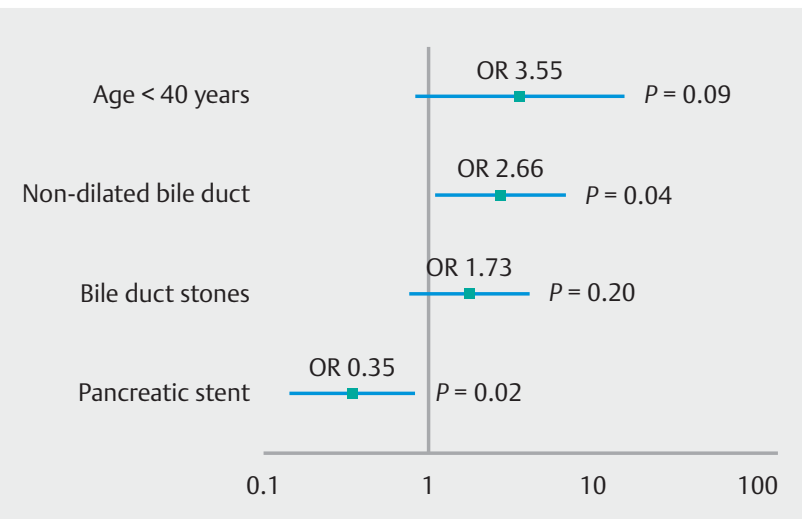

- Fig. 3 Forest plot showing risk factors for post-ERCP pancreatitis. Black squares indicate the odds ratio (OR), lines represent the $95 \%$ confidence interval.

risk factor (Terumo wire, OR 6.59; $95 \% \mathrm{Cl} 2.02$ - 21.52; $P<0.01$; difficult cannulation, OR 0.84; $95 \% \mathrm{Cl} 0.09-8.17 ; P=0.88$; pancreatic stent, OR $0.41 ; 95 \% \mathrm{Cl} 0.18-0.96 ; P=0.04)$. Furthermore, cannulating the pancreatic duct using a Terumo wire was associated with an even higher risk for PEP when cannulation of the papilla was not difficult (OR 6.59 vs. OR 4.89, $P=$ 0.81 ). Injection of contrast agent into the pancreatic duct was not an additional risk factor for PEP (contrast agent, OR 0.41; $95 \% \mathrm{Cl} 0.14-1.26) ; P=0.11$; Supplementary Table 1).

A final multivariable analysis, including the factors that were associated with an increased risk in the preceding analyses, again confirmed a non-dilated bile duct as an independent risk factor for PEP and the pancreatic stent as a protective measure ( Fig. 3).

\section{Outcome}

Median length of hospitalization after ERCP was 5.0 days (range, 1.0-99.0; IQR, 2.0-10.0) and was similar between the two groups (stent group, 5.5 days (range, 1.0-99.0; IQR 3.011.0 ) vs. no stent group, 5.0 days (range, 1.0-63.0; IQR, 2.0 9.3); $P=0.20)$. In the 87 patients who received a stent, $48 / 87$ (55.2\%) of the stents were removed endoscopically after a median of 5.0 days (range, $1.0-16.0 ;$ IQR, 4.0-6.0) and 34 (39.1\%) stents dislocated spontaneously. Three patients underwent pancreatic resection, one patient died, and one patient was transferred to another hospital. Spontaneous dislocation of the pancreatic stent was not associated with an increased risk of PEP in univariable analysis (OR 0.84; $95 \% \mathrm{Cl} 0.24-3.25$; $P=0.88)$

Among patients with PEP, median length of hospital stay after ERCP was 6.0 days (range,1.0-99.0; IQR, 3.0-10.0) and therefore 1 day longer than for patients without PEP (median, 5.0 days; range $1.0-63.0 ; \mathrm{IQR}, 2.0-10.0 ; P=0.28$ ). In the majority of patients, no procedure-related adverse events other than PEP occurred (139/167; 83.2\%; Table 2). Minor bleeding occurred in 24 of 167 patients (14.4\%) and perforation in three of 167 patients ( $1.8 \%$ ). One of the stents dislocated into the pancreatic duct; this patient died due to cholangiosepsis. There

\section{- Table 2 Complications of ERCP.}

\begin{tabular}{|l|r|r|c|}
\hline & Stent group & No stent group & P value \\
\hline PEP & $11 / 87(12.6 \%)$ & $20 / 80(25.0 \%)$ & $0.04^{1}$ \\
\hline Minor bleeding & $16 / 85(18.8 \%)$ & $8 / 78(10.2 \%)$ & 0.12 \\
\hline Duct Perforation & $3 / 85(3.5 \%)$ & $0 / 80(0.0 \%)$ & 0.25 \\
\hline \begin{tabular}{l} 
ERCP, endoscopic retrograde cholangiopancreatography. \\
\hline
\end{tabular} value $<0.05$
\end{tabular}

were no clinically relevant differences regarding adverse events between the two groups ( $\triangleright$ Table 2 ).

\section{Discussion}

Although PDS has been widely used for prevention of PEP in past years, the target population and optimal duration of stenting have not been well defined as of yet [18]. In this multicenter, randomized controlled trial, we found that PDS after inadvertent cannulation of the pancreatic duct in unselected patients reduced risk of PEP to $12.6 \%$ compared with $25.0 \%$ in the observational group. The strategy was associated with an absolute risk reduction of $12.4 \%$, equivalent to treating eight unselected patients to prevent one case of PEP. This study provides direct evidence to support the recommendation that PDS should be performed after inadvertent cannulation of the pancreatic duct during first-time ERCP in unselected patients.

Pancreatitis occurring after ERCP is a common and serious complication [19]. Several studies have identified patient- or procedure-related factors associated with an increased risk of PEP [20,21]. One consistent, patient-related risk is a non-dilated bile duct, which was confirmed in our cohort. Among the procedure-related risk factors, pancreatic cannulation has been identified as relevant for induction of PEP $[5,22,23]$. Mechanistically, triggering edema at the level of the duodenal papilla during the procedure very likely negatively impacts the outflow of pancreatic juice, thereby facilitating development of PEP through preserving drainage of the gland and emptying it from reactive pancreatic enzymes while another possible trigger is guidewire trauma of the pancreatic duct [24,25]. As we only included patients with inadvertent cannulation of the pancreatic duct, the frequency of PEP (18.6\%, 31/167 patients) among our unselected patients undergoing first-time ERCP was in the upper range when compared to previous studies [26]. Moreover, it has been reported that in first-time ERCPs, PEP rate is higher than in repeat ERCPs after endoscopic sphincteropapillotomy, which is in line with our study results $[22,27]$. To harmonize our study cohort, we randomized patients only in case of inadvertent cannulation, as intentional cannulation of the pancreatic duct most likely also increases risk of PEP. In addition, the increased rate of PEP in our study may also be due to the increased capture of complications in randomized controlled trials. Taking these criteria into account, the PEP rate in this study was acceptable and our study is in line with previous 
reports revealing pancreatic cannulation as a relevant procedure-related risk factor for PEP $[23,28]$.

Placement of a stent into the pancreatic duct has emerged as a reproducible tool to reduce PEP in high-risk cohorts [29, 30]. Interestingly, its use is not widespread, potentially due to the associated technical demands, risks of this procedure, and additional costs [31]. Pancreatic stent placement is - together with use of NSAIDs - the most rigorously studied prophylactic measure for prevention of PEP. Although this measure has decreased risk of mild, moderate, and severe, post-ERCP pancreatitis in patients both with a high and a low-to-mixed risk, it is not clear when and in which patients PDS should be performed [32]. Studies describing indications for pancreatic stenting during ERCP and demonstrating its efficacy in unselected patients are lacking. As such, our study is of imminent importance for a broad spectrum of hospitals as it demonstrates that pancreatic cannulation increases PEP and that this increase can be significantly reduced by placement of a pancreatic stent in an unselected patient cohort. Inadvertent cannulation of the pancreatic duct facilitates PDS, as the guidewire can be used immediately to place a pancreatic stent. This approach can further help to successfully cannulate the bile duct. Although we included only first-time ERCPs, in which the pancreatic duct was inadvertently cannulated, we cannot exclude that even in patients without accidental cannulation of the pancreatic duct, PDS is beneficial and averts development of PEP.

For post-cannulation prophylaxis of PEP, we placed a $5 \mathrm{Fr}$ pancreatic stent of at least $5 \mathrm{~cm}$ length that was removed after a median of 5 days if it had not spontaneously dislodged by that time. Some studies showed equal or even better effectiveness regarding risk reduction for PEP for smaller stents (3 Fr), arguing with fewer stent-induced ductal changes and less frequently need for endoscopic removal $[33,34]$. Disadvantages of small stents include the need for an additional wire (0.018inch guidewire for $3 \mathrm{Fr}$ stents) and more challenging stent placement, perhaps resulting in a higher rate of PEP. Accordingly, a recent meta-analysis and a large register-based study demonstrated better outcome for larger stents $[35,36]$. The reason for this might be better drainage ensuring decompression for larger stents and lower dislodgment rate for longer stents ensuring a prolonged effect. Indeed, early outward migration is a significant problem, particularly as it can lead to delayed-onset post-ERCP pancreatitis. Recent trials have shown that immediate removal or early outward migration of a PDS did not protect from PEP or showed delayed-onset of PEP compared with no PDS placement [37]. In our study, 34 of 87 stents (39\%) dislocated spontaneously. However, spontaneous dislocation of the pancreatic stent was not associated with an increased risk of PEP.

It remains unclear whether a pancreatic duct stent alone is more effective than indomethacin administration. Two clinical trials comparing the efficacies of PDS and indomethacin are ongoing (ClinicalTrials.gov Identifier: NCT02476279 and NCT02368795). Rectal NSAIDs reduced risk of PEP in averageand high-risk cohorts, especially when combined with PDS [38]. Head-to-head comparisons of rectal NSAIDs alone with PDS in high-risk cohorts are lacking so far. In a recent network meta-analysis, the combination of pancreatic duct stenting and NSAIDs did not further reduce the risk of post-ERCP pancreatitis compared with use of NSAIDs alone [38]. However, the meta-analysis has several drawbacks and consequently high-quality, randomized controlled trials will be needed to compare these two interventions.

\section{Limitations of the study}

Although this is a multicenter, prospective, randomized trial, the statistical power of hypothesis testing on several investigated risk factors, except for the primary outcome, was low (for example the subgroup age $<40$ contained only $10 / 167$ patients). Therefore, conclusions regarding these endpoints have to be confirmed through future studies and different populations.

\section{Conclusion}

In summary, prophylactic pancreatic duct stenting after inadvertent cannulation of the pancreatic duct is a safe and feasible procedure and significantly reduces the rate of post-ERCP pancreatitis in an unselected population. Thus, our study identifies a scenario in which PDS should be performed to prevent and alleviate PEP.

\section{Acknowledgements}

The authors thank the endoscopists other than the authors who performed the ERCPs for their collaboration, namely (in alphabetical order) M. Abdelhafez, M. Bajbouj, S. von Delius, C. Lübbert, O. Ebert, W. Huber, A. Meining, B. Neu, I. Ozola-Zālīte, D. Saur, J. Siveke, and A. Umgelter. We also thank Jörg Steiner, Texas A\&M University, for the detailed correction of language and grammar as well as critical review of the manuscript. We would like to recognize that the group of this multicenter study was formed during a course of Pancreas 2000, an educational and research program for tomorrow's pancreatologists in Europe.

This study is part of the doctoral theses of D. Haf and M. Schwab.

Competing interests

None

References

[1] Peery AF, Crockett SD, Barritt AS et al. Burden of Gastrointestinal, Liver, and Pancreatic Diseases in the United States. Gastroenterology 2015; 149: 1731 - 1741

[2] Phillip V, Steiner JM, Algul H. Early phase of acute pancreatitis: Assessment and management. World journal of gastrointestinal pathophysiology 2014; 5: $158-168$

[3] Talukdar R. Complications of ERCP. Best Pract Res Clin Gastroenterol 2016; 30: $793-805$ 
[4] Fogel EL, Eversman D, Jamidar P et al. Sphincter of Oddi dysfunction: pancreaticobiliary sphincterotomy with pancreatic stent placement has a lower rate of pancreatitis than biliary sphincterotomy alone. Endoscopy 2002; 34: 280-285

[5] Dumonceau JM, Andriulli A, Elmunzer B] et al. Prophylaxis of postERCP pancreatitis: European Society of Gastrointestinal Endoscopy (ESGE) Guideline - updated June 2014. Endoscopy 2014; 46: 799- 815

[6] Levenick JM, Gordon SR, Fadden LL et al. Rectal Indomethacin Does Not Prevent Post-ERCP Pancreatitis in Consecutive Patients. Gastroenterology 2016; 150: $911-917$

[7] Freeman ML, Kozarek RA. Take 2 indomethacin (suppositories) and call me in the morning? the role of nonsteroidal anti-inflammatory drugs in protection against post-endoscopic retrograde cholangiopancreatography pancreatitis Gastroenterology 2016; 150: 805-808

[8] Luo H, Zhao L, Leung J et al. Routine pre-procedural rectal indometacin versus selective post-procedural rectal indometacin to prevent pancreatitis in patients undergoing endoscopic retrograde cholangiopancreatography: a multicentre, single-blinded, randomised controlled trial. Lancet 2016; 387: 2293-2301

[9] Mazaki T, Mado K, Masuda $\mathrm{H}$ et al. Prophylactic pancreatic stent placement and post-ERCP pancreatitis: an updated meta-analysis. J Gastroenterol 2014; 49: 343-355

[10] Sofuni A, Maguchi H, Mukai T et al. Endoscopic pancreatic duct stents reduce the incidence of post-endoscopic retrograde cholangiopancreatography pancreatitis in high-risk patients. Clin Gastroenterol Hepatol 2011; 9: 851-858; quiz e110

[11] Gasslander T, Holmberg B, Permert J. Pancreas 2000 - a new concept for education and development in pancreatology. Pancreatology 2005; 5: 545-546

[12] Herreros de Tejada A, Calleja JL, Diaz G et al. Double-guidewire technique for difficult bile duct cannulation: a multicenter randomized, controlled trial. Gastrointest Endosc 2009; 70: 700 - 709

[13] Madacsy L, Kurucsai G, Fejes R et al. Prophylactic pancreas stenting followed by needle-knife fistulotomy in patients with sphincter of Oddi dysfunction and difficult cannulation: new method to prevent post-ERCP pancreatitis. Dig Endosc 2009; 21: 8-13

[14] Banks PA, Bollen TL, Dervenis C et al. Classification of acute pancreatitis-2012: revision of the Atlanta classification and definitions by international consensus. Gut 2013; 62: 102-111

[15] Harrell FE. Regression Modeling Strategies. New York, NY: Springer; 2001

[16] Kay R. Statistical principles for clinical trials. J Int Med Res 1998; 26 : $57-65$

[17] Bender R. Calculating confidence intervals for the number needed to treat. Control Clin Trials 2001; 22: $102-110$

[18] Freeman ML. Preventing Post-ERCP pancreatitis: Update 2016. Curr Treat Options Gastroenterol 2016; 14: 340 - 347

[19] Kochar B, Akshintala VS, Afghani E et al. Incidence, severity, and mortality of post-ERCP pancreatitis: a systematic review by using randomized, controlled trials. Gastrointest Endosc 2015; 81: $143-$ 149 e 149

[20] Ding X, Zhang F, Wang Y. Risk factors for post-ERCP pancreatitis: A systematic review and meta-analysis. Surgeon 2015; 13: 218-229

[21] Freeman ML, DiSario JA, Nelson DB et al. Risk factors for post-ERCP pancreatitis: a prospective, multicenter study. Gastrointest Endosc 2001; 54: $425-434$
[22] Phillip V, Schwab M, Haf D et al. Identification of risk factors for postendoscopic retrograde cholangiopancreatography pancreatitis in a high volume center. PLoS One 2017; 12: e0177874

[23] Nakai $\mathrm{Y}$, Isayama $\mathrm{H}$, Sasahira $\mathrm{N}$ et al. Risk factors for post-ERCP pancreatitis in wire-guided cannulation for therapeutic biliary ERCP. Gastrointest Endosc 2015; 81: 119-126

[24] Buxbaum J, Leonor P, Tung J et al. Randomized trial of endoscopistcontrolled vs. assistant-controlled wire-guided cannulation of the bile duct. Am J Gastroenterol 2016; 111: 1841 - 1847

[25] Srinivasan I, Freeman ML. Editorial: Guidewire Trauma: A key component of post-ercp pancreatitis that is best controlled by the endoscopist. Am J Gastroenterol 2016; 111: 1848 - 1850

[26] Testoni PA. Why the incidence of post-ERCP pancreatitis varies considerably? Factors affecting the diagnosis and the incidence of this complication JOP 2002; 3: 195-201

[27] Matsubayashi H, Fukutomi A, Kanemoto $\mathrm{H}$ et al. Risk of pancreatitis after endoscopic retrograde cholangiopancreatography and endoscopic biliary drainage. HPB 2009; 11: $222-228$

[28] Parekh PJ, Majithia R, Sikka SK et al. The "scope" of post-ERCP pancreatitis. Mayo Clin Proc 2017; 92: 434-448

[29] Shi QQ, Ning XY, Zhan LL et al. Placement of prophylactic pancreatic stents to prevent post-endoscopic retrograde cholangiopancreatography pancreatitis in high-risk patients: a meta-analysis. World J Gastroenterol 2014; 20: 7040 - 7048

[30] Lee TH, Moon JH, Choi H] et al. Prophylactic temporary 3F pancreatic duct stent to prevent post-ERCP pancreatitis in patients with a difficult biliary cannulation: a multicenter, prospective, randomized study. Gastrointest Endosc 2012; 76: 578-585

[31] Dumonceau JM, Rigaux J, Kahaleh M et al. Prophylaxis of post-ERCP pancreatitis: a practice survey. Gastrointest Endosc 2010; 71: 934 939

[32] Freeman ML. Use of Prophylactic Pancreatic Stents for the Prevention of Post-ERCP Pancreatitis. Gastroenterol Hepatol (NY) 2015; 11: $420-422$

[33] Pahk A, Rigaux J, Poreddy V et al. Prophylactic pancreatic stents: does size matter? A comparison of 4-Fr and 5-Fr stents in reference to postERCP pancreatitis and migration rate Dig Dis Sci 2011; 56: 3058 3064

[34] Rashdan A, Fogel EL, McHenry L et al. Improved stent characteristics for prophylaxis of post-ERCP pancreatitis. Clin Gastroenterol Hepatol 2004; 2: $322-329$

[35] Afghani E, Akshintala VS, Khashab MA et al. 5-Fr vs. 3-Fr pancreatic stents for the prevention of post-ERCP pancreatitis in high-risk patients: a systematic review and network meta-analysis. Endoscopy 2014; 46: $573-580$

[36] Olsson G, Lubbe J, Arnelo U et al. The impact of prophylactic pancreatic stenting on post-ERCP pancreatitis: A nationwide, register-based study. United European Gastroenterol J 2017; 5: 111-118

[37] Moffatt DC, Pradermchai K, Avula $\mathrm{H}$ et al. Moderate and severe postendoscopic retrograde cholangiopancreatography pancreatitis despite prophylactic pancreatic stent placement: the effect of early prophylactic pancreatic stent dislodgement. Can J Gastroenterol 2011; 25: $215-219$

[38] Akbar A, Abu Dayyeh BK, Baron TH et al. Rectal nonsteroidal anti-inflammatory drugs are superior to pancreatic duct stents in preventing pancreatitis after endoscopic retrograde cholangiopancreatography: a network meta-analysis. Clin Gastroenterol Hepatol 2013; 11: $778-783$ 
- Supplementary Table 1 Univariable analysis of risk factors for post-ERCP pancreatitis.

\begin{tabular}{|c|c|c|}
\hline Risk factor & Odds ratio (Cl $95 \%)$ & $P$ value \\
\hline \multicolumn{3}{|l|}{ Patient related } \\
\hline Female gender & $1.21(0.55-2.70)$ & 0.64 \\
\hline Age, years & $1.00(0.98-1.03)$ & 0.80 \\
\hline Age $<40$ years $(n=10)$ & $3.21(0.85-12.15)$ & 0.07 \\
\hline Body weight, $\mathrm{Kg}$ & $0.99(0.96-1.02)$ & 0.49 \\
\hline Body height, cm & $1.00(0.96-1.06)$ & 0.89 \\
\hline $\mathrm{BMI}, \mathrm{kg} / \mathrm{m}^{2}$ & $0.95(0.85-1.06)$ & 0.37 \\
\hline $\mathrm{BMI}>25 \mathrm{~kg} / \mathrm{m}^{2}(\mathrm{n}=74)$ & $0.76(0.32-1.83)$ & 0.55 \\
\hline Bile duct diameter, mm & $0.92(0.85-1.00)$ & 0.06 \\
\hline Non dilated bile duct diameter $(<8 \mathrm{~mm})(\mathrm{n}=39)$ & $2.00(0.86-4.67)$ & 0.10 \\
\hline Serum bilirubin, mg/dL & $0.94(0.86-1.02)$ & 0.11 \\
\hline Normal serum bilirubin $(<1.2 \mathrm{mg} / \mathrm{dL})(\mathrm{n}=51)$ & $1.81(0.81-4.05)$ & 0.15 \\
\hline Previous acute pancreatitis $(n=14)$ & $0.71(0.15-3.36)$ & 1.00 \\
\hline Chronic pancreatitis $(n=9)$ & $0.53(0.06-4.43)$ & 1.00 \\
\hline Status post cholecystectomy $(n=32)$ & $1.02(0.38-2.73)$ & 0.98 \\
\hline Juxtapapillary diverticulum ( $\mathrm{n}=22)$ & $0.97(0.30-3.10)$ & 1.00 \\
\hline \multicolumn{3}{|l|}{ Diagnosis related } \\
\hline Bile duct stones $(n=69)$ & $1.96(0.89-4.31)$ & 0.09 \\
\hline Jaundice of unknown origin $(n=10)$ & $0.93(0.88-0.97)$ & 0.21 \\
\hline Benign bile duct stricture $(n=24)$ & $0.86(0.27-2.72)$ & 1.00 \\
\hline Malignant bile duct stricture $(n=42)$ & $1.04(0.43-2.55)$ & 0.93 \\
\hline Unknown bile duct stricture $(n=16)$ & $0.60(0.13-2.80)$ & 0.74 \\
\hline Any bile duct stricture $(n=82)$ & $0.82(0.38-1.80)$ & 0.63 \\
\hline \multicolumn{3}{|l|}{ Procedure related } \\
\hline Urgent indication for $\operatorname{ERCP}(n=30)$ & $0.60(0.19-1.86)$ & 0.37 \\
\hline Difficult cannulation of papilla $(n=58)$ & $0.87(0.38-2.00)$ & 0.75 \\
\hline Huibregtese/cannula/bile duct ( $n=67$ ) & $3.44(1.52-7.79)$ & $<0.01^{1}$ \\
\hline Terumo/bile duct $(n=84)$ & $4.34(1.75-10.76)$ & $<0.01^{1}$ \\
\hline Teflon/bile duct $(n=76)$ & $3.09(1.35-7.07)$ & $0.01^{1}$ \\
\hline Short wire/bile duct $(n=44)$ & $0.62(0.45-1.63)$ & 0.33 \\
\hline Any wire/bile duct $(n=143)$ & $1.16(0.37-3.68)$ & 0.80 \\
\hline Bile duct stent $(n=88)$ & $2.16(0.95-4.93)$ & 0.06 \\
\hline Huibregtese/cannula/pancreatic duct ( $\mathrm{n}=15$ ) & $0.65(0.14-3.05)$ & 0.74 \\
\hline Terumo/pancreatic duct $(n=93)$ & $5.36(1.94-14.77)$ & $<0.001^{1}$ \\
\hline Teflon/pancreatic duct $(n=47)$ & $1.06(0.45-2.50)$ & 0.90 \\
\hline Short wire/pancreatic duct $(n=54)$ & $0.55(0.22-1.38)$ & 0.20 \\
\hline Papillotome/pancreatic duct $(n=91)$ & $2.88(1.20-6.88)$ & $0.02^{1}$ \\
\hline Any wire/pancreatic duct ( $n=159$ ) & $1.63(0.19-13.74)$ & 0.65 \\
\hline Contrast agent/pancreatic duct $(n=40)$ & $0.41(0.14-1.26)$ & 0.11 \\
\hline
\end{tabular}


- Supplementary Table 1 (Continuation)

\begin{tabular}{|c|c|c|}
\hline Risk factor & Odds ratio (Cl $95 \%)$ & $P$ value \\
\hline Acinarization/pancreatic duct $(n=4)$ & $0.97(0.94-1.00)$ & 1.00 \\
\hline Pancreatic stent $(n=87)$ & $0.43(0.19-0.98)$ & $0.04^{1}$ \\
\hline Transpancreatic papillotomy $(n=68)$ & $0.90(0.41-2.01)$ & 0.80 \\
\hline Needle knife $(n=21)$ & $0.19(0.03-1.50)$ & 0.08 \\
\hline Papillotomy/cutting ( $\mathrm{n}=54$ ) & $0.68(0.28-1.64)$ & 0.390 \\
\hline Papillotomy/blended $(n=5)$ & $0.96(0.93-1.00)$ & 0.59 \\
\hline Papillotomy/endocut $(\mathrm{n}=95)$ & $1.48(0.66-3.32)$ & 0.34 \\
\hline Complete stone removal $(\mathrm{n}=38)$ & $0.89(0.30-2.68)$ & 0.84 \\
\hline No or incomplete stone removal $(n=30)$ & $1.21(0.40-3.64)$ & 0.73 \\
\hline Dormia basket/bile duct $(n=49)$ & $1.69(0.75-3.82)$ & 0.20 \\
\hline Balloon/bile duct $(n=15)$ & $1.18(0.31-4.49)$ & 0.81 \\
\hline \multicolumn{3}{|l|}{ Complications } \\
\hline Hemorrhage $(n=24)$ & $1.26(0.43-3.71)$ & 0.77 \\
\hline Perforation $(n=3)$ & $2.36(0.21-26.91)$ & 0.45 \\
\hline No complication $(n=139)$ & $0.73(0.27-2.00)$ & 0.54 \\
\hline \multicolumn{3}{|l|}{ Anesthesia related } \\
\hline Midazolam, mg & $0.71(0.34-1.48)$ & 0.37 \\
\hline Fentanyl, mg & $0.55(0.01-64.82)$ & 0.80 \\
\hline Disoprivan, mg & $1.00(1.00-1.00)$ & 0.134 \\
\hline $\begin{array}{l}\text { BMI, Body mass index; ERCP, endoscopic retro } \\
{ }_{1} P \text { value }<0.05\end{array}$ & & \\
\hline
\end{tabular}

